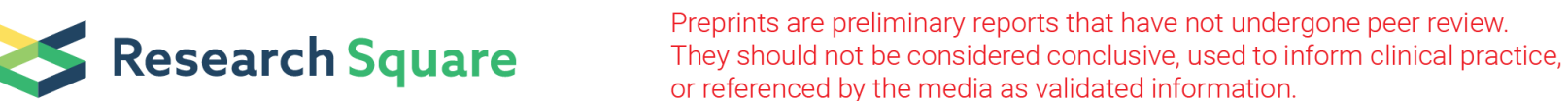

\section{ADCY2, ADCY5, and GRIA1 are the key genes of CAMP signaling pathway to participate in osteoporotic peripheral and spinal fracture after the manipulation of Wnt signaling}

\section{Xiaohua Zuo}

Nanjing First Hospital

Changdong Zhou

Huaian City Second People's Hospital

Xuepiao Zhu

Huaian City Second People's Hospital

Dan Liu

Huaian City Second People's Hospital

Yan Wang ( $\sim$ WY335556@163.com )

The Second People's Hospital of Lianyungang Affiliated to Jiangsu University

Hongguang Bao ( $D$ BHG23356@163.com )

Nanjing First Hospital

Kai Zhang

Huaian City Second People's Hospital

Yong Zhang

Huaian City Second People's Hospital

\section{Research}

Keywords: Spinal Fractures; Wnt Signaling Pathway; cAMP signaling; Osteoporotic Fractures; Adenylate Cyclase

Posted Date: April 29th, 2020

DOI: https://doi.org/10.21203/rs.3.rs-25264/v1

License: (c) (1) This work is licensed under a Creative Commons Attribution 4.0 International License.

Read Full License 
Version of Record: A version of this preprint was published at Archives of Medical Science on April 15th, 2021. See the published version at https://doi.org/10.5114/aoms/125210. 


\section{Abstract \\ Background}

Osteoporotic peripheral and spinal fracture, characterized by high morbidity and mortality, has become a health burden for the aging population. The inactivation of the Wnt signaling has been proved to promote osteoporotic fractures. Our study is to identify the key genes, miRNAs, and pathways that possibly lead to osteoporosis and osteoporotic peripheral and spinal fracture after the aberrant activation or mutation of Wnt signaling pathway.

\section{Methods}

Impute R package was used to screen out the differently expressed genes (DEGs) and differently expressed miRNAs in GEO datasets. STRING and Metascape were used to construct protein-protein interactions (PPI) network, gene ontology (GO) enrichment and pathway enrichment.

\section{Results}

562 DEGs were screened out using Impute R package, and a PPI network involving the 562 DEGs was constructed using STRING and Metascape. GO enrichment and pathway enrichment showed that the 562 DEGs were associated with membrane protein-related signaling pathways. Then, 75 genes between the target genes of miR-18a-3p and 562 DEGs were overlapped using Venny 2.1.0. Finally, the cAMP signaling pathway was identified as the key pathway, whilst ADCY2, ADCY5, and GRIA1 were identified the key genes that possibly participate in osteoporotic peripheral and spinal fracture after the manipulation of Wnt signaling pathway.

\section{Conclusions}

The results demonstrated that $A D C Y 2, A D C Y 5$, and GRIA1 were the key genes to regulate the CAMP signaling pathway in osteoporotic peripheral and spinal fracture after abnormal Wnt signaling.

\section{Background}

Osteoporosis is a common disorder caused by the imbalance between osteoblastic bone formation and osteoclastic bone resorption (1). Age is proportional to the risk of development of osteoporosis (2). Due to the absence of estrogen, women after menopause were at the highest risk of getting osteoporosis according to a previous study (3). Certainly, the absence of androgenic hormones could also cause osteoporosis in men (4). Besides, other factors also led to osteoporosis such as metabolic diseases, anorexia nervosa, thyroid and renal dysfunctions or dietary as well as lifestyle habits like low calcium intake or immobilization (5). 
Osteoporosis can lead to a reduction in bone mass, deterioration in bone microarchitecture, susceptibility to skeletal fragility, and increased risk of fracture (6). The patients suffering osteoporotic fractures particularly spinal fracture are characterized by high morbidity and mortality so that the quality of life is significantly decreased. It has been reported that up to one-third of patients will sustain a new fracture within 5 years after the initial fracture (7). Although anti-osteoporosis drugs reduce the risk of osteoporotic fractures by $20-70 \%$ in clinical trials depending on the drug and fracture type, the persistence with osteoporosis therapy is poor, and the one-year persistence ranges from 18 to $78 \%$ between studies in the real-world (8-13). In fact, the postmenopausal women over age 55 are sensitive to the osteoporosis-related spinal fracture (14). Therefore, understanding the key mechanism of osteoporotic fractures is crucial for treating spinal fracture.

Wingless-related integration site (Wnt)/ $\beta$-catenin signaling pathway is the key pathway of bone metabolism to regulate bone mass. The defective Wnt signaling causes several monogenic skeletal disorders such as osteoporosis-pseudoglioma syndrome, van Buchem disease, and sclerosteosis (1517). For example, WNT7B enhanced the ability of bone formation by increasing osteoblast activity to increase bone mass (18). Glucocorticoids depressed bone formation by inhibiting Wnt/ $\beta$-catenin signaling pathway (19). Laine CM et al. found that the mutation of Wnt1 could decrease the activity of the $\mathrm{Wnt} / \beta$-catenin signaling pathway in bone leading to the decrease of the number of bone cells, damage of bone formation, low bone mass, and skeletal fragility (20). If the skeletal fragility happened in vertebrae, the spinal fracture might be caused by simple movements such as coughing or sneezing. Mäkitie RE et al. also proved that impaired WNT/ $\beta$-catenin signaling progressively changed the the spinal structures, which increased the risk of compression fractures especially after the age of 50 (21).

In this study, the expression profiles of mRNA and miRNA after manipulation of Wnt signaling were obtained from GEO DataSets. Then, the bioinformatic analysis including GO enrichment, KEGG enrichment, Reactome pathway, and PPI network was performed to analyze the key pathway, miRNAs, and genes after the mutation of the Wnt signaling pathway in osteoporosis and osteoporotic fractures. In the long term, our study should contribute to the treatment of osteoporotic fractures especially peripheral and spinal fracture.

\section{Material And Methods}

\section{Data collection and array data analysis}

Two expression profile data sets, GSE34747 and GSE103473, relating to osteoporosis and peripheral and spinal fracture were downloaded from GEO database (https://www.ncbi.nlm.nih.gov/gds/). The differently expressed genes (DEGs) of GSE34747 between Wnt activation samples $(n=3)$ and normal samples $(n=3)$ were identified using impute R package. The differently expressed miRNAs of GSE103473 between Wnt1 mutation samples $(n=12)$ and normal samples $(n=12)$ were identified using impute $R$ package. The DEGs and differently expressed miRNAs were selected with the log |fold change| value $\geq 1$ 


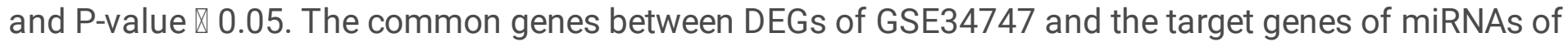
GSE103473 were overlapped by Venny 2.1.0.

\section{The construction and analysis of protein-protein interactions (PPI) network}

To construct the PPI network, DEGs were uploaded to STRING (https://string-db.org/) and Metascape (http://metascape.org/gp/index.html\#/main/step1), respectively. STRING was an online tool to predict and visualize the PPI which includes direct and indirect associations. Metascape was an online gene annotation and analysis tool, which could analyze and visualize the PPI network. The analysis of PPI by Metascape algorithm depends on BioGrid, InWeb_IM, OmniPath databases. Molecular Complex Detection (MCODE) was applied to identify connected network components.

\section{Gene ontology (GO) enrichment and pathway enrichment analysis}

GO enrichment of DEGs including biological process, molecular function, and cellular component was analyzed using STRING and Metascape. Reactome pathway database was a relational database of signaling and metabolic molecules. STRING PPI network construction was performed to analyze the Reactome pathways of DEGs. Kyoto Encyclopedia of Genes and Genomes (KEGG) pathways containing the information of the network of genes or molecules were also analyzed by STRING and Metascape.

\section{Results}

\section{GO enrichment and Reactome pathway enrichment of 562 DEGs using STRING}

Wnt signaling was associated with osteoporosis and could be activated by lithium. GSE34747 including the LiCl-stimulated samples (Wnt activation samples) and normal samples was analyzed by R software. Hierarchical clustering analysis showed that the datasets were well clustered: most genes in Wnt activation samples and normal samples tended to be grouped in two clusters, while there were limited overlapped parts (Fig.1A). For the functional enrichment analysis by STRING, 562 DEGs were finally selected with the fold change value $\geq 1$ and P-value $₫ 0.05$ and displayed the complicated PPI network (Fig.1B). GO enrichment showed that the biological process of 562 DEGs was associated with the chemical stimulus, the molecular function of 562 DEGs was associated with receptor activity, and cellular component was associated with membrane (Fig.1C). Meanwhile, Reactome pathway analysis revealed that GPCR was the key signaling which had been proved to be related to osteoporosis. These results demonstrated that 562 DEGs might be associated with membrane protein-related signaling pathways.

\section{The analysis of process enrichment, pathway enrichment, and PPI network of DEGs using Metascape}

To further identify the function of 562 DEGs, another algorithm, Metascape, was used to analyze and visualize the key processes and pathways. As shown in Fig.2A, the calcium signaling pathway which was consistent with the GO analysis results of STRING, was the key pathway. In addition, the PPI network constructed by Metascape displayed 5 MCODEs (Fig.2B). The top 3 MCODEs were calcium signaling 
pathway, cAMP signaling pathway, and anterograde trans-synaptic signaling. The results of Metascape identified that the calcium signaling pathway and cAMP signaling pathway were the key pathways.

\section{The identification of overlapping genes between the target genes of miRNAs and DEGs}

GSE103473 was the miRNA profile of spinal fracture involving the Wnt1 mutation samples and normal samples. Hierarchical clustering analysis showed that most miRNAs in Wnt mutation samples and normal samples tended to be grouped in two clusters, while there was some degree of overlapping (Fig.3A). miR-34a-5p, miR-22-3p, miR-143-5p, miR-18a-3p, miR-31-5p, and miR-223-3p were the top 6 differently expressed miRNAs of GSE103473. Venny 2.1.0 was then used to select the overlapping genes between the target genes of the top 6 differently expressed miRNAs and the formerly identified 562 DEGs of GSE34747. Due to the most overlapping genes in miR-18a-3p, miR-18a-3p and overlapping 75 genes were screened out, which was associated with osteoporosis and spinal fracture (Fig.3B-G).

\section{The identification of key genes using STRING and Metascape}

To explore the biological functions of 75 genes involving in osteoporosis and peripheral and spinal fracture, STRING was first used to construct the PPI network of the 75 genes. The PPI network analysis showed that ADCY5, ADCY2, MLLT4, and GRIA1 were the genes associated with the CAMP signaling pathway (Fig.4A). Similar to the result of STRING, process and pathway analysis by Metascape also revealed that the cAMP signaling pathway was the key pathway (Fig.4B). Compared the results of STRING with Metascape, ADCY2, ADCY5, and GRIA1 were identified as the significant genes that were associated with the CAMP signaling pathway and possibly participate in osteoporosis and spinal fracture when Wnt signaling was manipulated (Fig.4C).

\section{Discussion}

Spinal fracture induced by osteoporosis has become a health burden of the aging population, especially in postmenopausal women over 55 . The activation of the Wnt/ $\beta$-catenin signaling pathway has been proved to prevent osteoblast and osteocyte apoptosis so it acts as a negative role in osteoporosis (19). In this study, the GO analysis and Reactome pathway analysis revealed that 562 DEGs might be associated with membrane protein-related signaling pathways especially calcium signaling pathway and cAMP signaling pathway. By analyzing the miRNA microarray, the 75 target genes of miR-18a-3p were screened out for further PPI network construction and GO term enrichments. Both STRING and Metascape enrichments identified that the CAMP signaling pathway was a crucial pathway. By comparing the results of STRING and Metascape, ADCY2, ADCY5, and GRIA1 were thought to be the key genes involving in osteoporosis and peripheral and spinal fracture after the manipulation of Wnt signaling.

Wnt/ $\beta$-catenin signaling pathway promoted the regeneration of osseous tissue by stimulating proliferation and differentiation of osteoblasts $(22,23)$. The Wnt proteins are secreted glycoproteins, which can stimulate the signaling pathway by binding to LRP5/6 and co-receptor Fizzled (24). Then, the receptors including Dsh, Axin, and APC can inhibit the activity of glycogen synthase kinase 3 (GSK3) to 
prevent the phosphorylation of $\beta$-catenin (25). The phosphorylation of $\beta$-catenin results in the degradation of $\beta$-catenin so that the Wnt/ $\beta$-catenin is inactivated. $\mathrm{Kim} \mathrm{JH}$ et al. found that $\beta$-catenin expression in bone tissues from patients suffering from osteoporotic fractures was reduced, indicating that the decrease of $\beta$-catenin could cause osteoporotic fractures (23). In our study, impaired Wnt signaling led to significant miR-18a-3p upregulation that possibly participated in osteoporosis and peripheral and spinal fracture. 75 genes that were both target genes of miR-18a-3p and DEGs caused by proactive Wnt activation went through STRING and Metascape interrogation, which demonstrated that the CAMP signaling pathway was the key pathway that might be associated with Wnt activation or mutation. We then concluded that the activation or inactivation of the Wnt signaling pathway could affect the CAMP signaling, which therefore affecting osteoporosis, and peripheral and spinal fracture processes.

Cyclic 3',5'-adenosine monophosphate (cAMP) is an important second messenger in bone homeostasis, which acts as a prominent role in determining the fate of cells. The intracellular cAMP level could be elevated by activating the G-protein-coupled receptor (GPCR) that was the major mediator of bone remodeling by inhibiting osteoblasts apoptosis and enhancing osteoblasts differentiation (26). In the present study, the Reactome pathways analysis exhibited that signaling by GPCR was closely associated with Wnt activation, which was consistent with the result of 75 overlapping genes' enrichment analysis. cAMP has been proved to inhibit osteoblast proliferation by suppressing the MAP kinase pathway (27). Weivoda MM et al. investigated the relationship between cAMP and Wnt pathway and found that Wnt3a suppressed osteoclast differentiation by activating the cAMP/PKA pathway (28). Together with the previous studies and our bioinformatic analysis, we believed that the CAMP signaling pathway was closely associated with the Wnt signaling, and thus may participate in osteoporotic fractures.

Adenylate cyclase 2 ( $A D C Y 2$ ) encodes a member of the adenylate cyclase family, which can catalyze the formation of CAMP (29). Adenylate cyclase 5 (ADCY5) encodes a member of the membrane-bound adenylyl cyclase enzymes which catalyze the formation of cAMP under the stimulation of G-protein signaling (29-31). Both ADCY2 and ADCY5 have not been investigated in osteoporotic fractures. Glutamate lonotropic Receptor AMPA Type Subunit 1 (GRIA1) belonging to a family of AMPA receptors only have been proved to be a tumor suppressor gene in human osteosarcoma (32). Therefore, the identified genes ADCY2, ADCY5, and GRIA1 are of high significance in regards to osteoporosis and potentially peripheral and spinal fracture, thus need to be further studied, which may provide the new therapeutic strategies for osteoporotic fractures.

\section{Conclusion}

In conclusion, we identified that the cAMP signaling pathway was associated with the activation or inactivation of the Wnt signaling pathway in osteoporotic fractures. Meanwhile, ADCY2, ADCY5, and GRIA1 were associated with osteoporotic fractures involving the Wnt pathway and cAMP pathway due to the little studies on these genes in osteoporotic fractures. Our findings might provide novel therapeutic strategies for osteoporotic fractures. 


\section{Declarations}

\section{Ethics approval and consent to participate}

Not applicable.

\section{Consent for publication}

Not applicable.

\section{Availability of data and materials}

The datasets used during the current study are available from the corresponding author on reasonable request.

\section{Competing interests}

The authors declare that there is no conflict of interests.

\section{Funding}

No funding was received.

\section{Author contributions}

YW and HGB designed the study. XHZ and CDZ performed most of the experiments. XPZ and DL collected the data. $\mathrm{KZ}$ and $\mathrm{YZ}$ wrote the paper. All authors read and approved the final manuscript.

\section{Acknowledgements}

Not applicable.

\section{References}

1. Teitelbaum SL. Bone resorption by osteoclasts. Science. 2000;289(5484):1504-8.

2. Wright NC, Looker AC, Saag KG, Curtis JR, Delzell ES, Randall S, et al. The recent prevalence of osteoporosis and low bone mass in the United States based on bone mineral density at the femoral neck or lumbar spine. Journal of bone and mineral research : the official journal of the American Society for Bone and Mineral Research. 2014;29(11):2520-6.

3. Foessl I, Kotzbeck P, Obermayer-Pietsch B. miRNAs as novel biomarkers for bone related diseases. Journal of Laboratory and Precision Medicine. 2019;4.

4. Golds G, Houdek D, Arnason T. Male Hypogonadism and Osteoporosis: The Effects, Clinical Consequences, and Treatment of Testosterone Deficiency in Bone Health. International journal of endocrinology. 2017;2017:4602129. 
5. Kanis JA, McCloskey EV. Risk factors in osteoporosis. Maturitas. 1998;30(3):229-33.

6. Keen R. Osteoporosis: strategies for prevention and management. Best practice \& research Clinical rheumatology. 2007;21(1):109-22.

7. Klop C, Welsing PM, Elders PJ, Overbeek JA, Souverein PC, Burden AM, et al. Long-term persistence with anti-osteoporosis drugs after fracture. Osteoporosis international : a journal established as result of cooperation between the European Foundation for Osteoporosis and the National Osteoporosis Foundation of the USA. 2015;26(6):1831-40.

8. Wells GA, Cranney A, Peterson J, Boucher M, Shea B, Robinson V, et al. Alendronate for the primary and secondary prevention of osteoporotic fractures in postmenopausal women. The Cochrane database of systematic reviews. 2008(1):CD001155.

9. Wells G, Cranney A, Peterson J, Boucher M, Shea B, Robinson V, et al. Risedronate for the primary and secondary prevention of osteoporotic fractures in postmenopausal women. The Cochrane database of systematic reviews. 2008(1):CD004523.

10. Netelenbos JC, Geusens PP, Ypma G, Buijs SJ. Adherence and profile of non-persistence in patients treated for osteoporosis-a large-scale, long-term retrospective study in The Netherlands. Osteoporosis international : a journal established as result of cooperation between the European Foundation for Osteoporosis and the National Osteoporosis Foundation of the USA. 2011;22(5):1537-46.

11. Li L, Roddam A, Gitlin M, Taylor A, Shepherd S, Shearer A, et al. Persistence with osteoporosis medications among postmenopausal women in the UK General Practice Research Database. Menopause. 2012;19(1):33-40.

12. van Boven JF, de Boer PT, Postma MJ, Vegter S. Persistence with osteoporosis medication among newly-treated osteoporotic patients. Journal of bone and mineral metabolism. 2013;31(5):562-70.

13. Confavreux CB, Canoui-Poitrine F, Schott AM, Ambrosi V, Tainturier V, Chapurlat RD. Persistence at 1 year of oral antiosteoporotic drugs: a prospective study in a comprehensive health insurance database. European journal of endocrinology. 2012;166(4):735-41.

14. Evans AJ, Jensen ME, Kip KE, DeNardo AJ, Lawler GJ, Negin GA, et al. Vertebral compression fractures: pain reduction and improvement in functional mobility after percutaneous polymethylmethacrylate vertebroplasty retrospective report of 245 cases. Radiology. 2003;226(2):366-72.

15. Gong Y, Slee RB, Fukai N, Rawadi G, Roman-Roman S, Reginato AM, et al. LDL receptor-related protein 5 (LRP5) affects bone accrual and eye development. Cell. 2001;107(4):513-23.

16. Loots GG, Kneissel M, Keller H, Baptist M, Chang J, Collette NM, et al. Genomic deletion of a longrange bone enhancer misregulates sclerostin in Van Buchem disease. Genome research. 2005;15(7):928-35.

17. Balemans W, Ebeling M, Patel N, Van Hul E, Olson P, Dioszegi M, et al. Increased bone density in sclerosteosis is due to the deficiency of a novel secreted protein (SOST). Human molecular genetics. 2001;10(5):537-43. 
18. Chen J, Tu X, Esen E, Joeng KS, Lin C, Arbeit JM, et al. WNT7B promotes bone formation in part through mTORC1. PLoS genetics. 2014;10(1):e1004145.

19. Guanabens N, Gifre L, Peris P. The role of Wnt signaling and sclerostin in the pathogenesis of glucocorticoid-induced osteoporosis. Current osteoporosis reports. 2014;12(1):90-7.

20. Jacobs S, Hansen F, Kasl S, Ostfeld A, Berkman L, Kim K. Anxiety disorders during acute bereavement: risk and risk factors. The Journal of clinical psychiatry. 1990;51(7):269-74.

21. Makitie RE, Niinimaki T, Nieminen MT, Schalin-Jantti C, Niinimaki J, Makitie O. Impaired WNT signaling and the spine-Heterozygous WNT1 mutation causes severe age-related spinal pathology. Bone. 2017;101:3-9.

22. Collins JN, Kirby BJ, Woodrow JP, Gagel RF, Rosen CJ, Sims NA, et al. Lactating Ctcgrp nulls lose twice the normal bone mineral content due to fewer osteoblasts and more osteoclasts, whereas bone mass is fully restored after weaning in association with up-regulation of Wnt signaling and other novel genes. Endocrinology. 2013;154(4):1400-13.

23. Kim JH, Liu X, Wang J, Chen X, Zhang H, Kim SH, et al. Wnt signaling in bone formation and its therapeutic potential for bone diseases. Therapeutic advances in musculoskeletal disease. 2013;5(1):13-31.

24. Suen PK, Qin L. Sclerostin, an emerging therapeutic target for treating osteoporosis and osteoporotic fracture: A general review. Journal of orthopaedic translation. 2016;4:1-13.

25. Cong F, Schweizer L, Varmus H. Wnt signals across the plasma membrane to activate the betacatenin pathway by forming oligomers containing its receptors, Frizzled and LRP. Development. 2004;131(20):5103-15.

26. Kim JM, Choi JS, Kim YH, Jin SH, Lim S, Jang HJ, et al. An activator of the cAMP/PKA/CREB pathway promotes osteogenesis from human mesenchymal stem cells. Journal of cellular physiology. 2013;228(3):617-26.

27. Chaudhary LR, Avioli LV. Identification and activation of mitogen-activated protein (MAP) kinase in normal human osteoblastic and bone marrow stromal cells: attenuation of MAP kinase activation by CAMP, parathyroid hormone and forskolin. Molecular and cellular biochemistry. 1998;178(1-2):59-68.

28. Weivoda MM, Ruan M, Hachfeld CM, Pederson L, Howe A, Davey RA, et al. Wnt Signaling Inhibits Osteoclast Differentiation by Activating Canonical and Noncanonical cAMP/PKA Pathways. Journal of bone and mineral research : the official journal of the American Society for Bone and Mineral Research. 2016;31(1):65-75.

29. Ding Q, Gros R, Gray ID, Taussig R, Ferguson SS, Feldman RD. Raf kinase activation of adenylyl cyclases: isoform-selective regulation. Molecular pharmacology. 2004;66(4):921-8.

30. Brand CS, Sadana R, Malik S, Smrcka AV, Dessauer CW. Adenylyl Cyclase 5 Regulation by Gbetagamma Involves Isoform-Specific Use of Multiple Interaction Sites. Molecular pharmacology. 2015;88(4):758-67.

31. Chen YZ, Friedman JR, Chen DH, Chan GC, Bloss CS, Hisama FM, et al. Gain-of-function ADCY5 mutations in familial dyskinesia with facial myokymia. Annals of neurology. 2014;75(4):542-9. 
32. Bernardini G, Laschi M, Serchi T, Spreafico A, Botta M, Schenone S, et al. Proteomics and phosphoproteomics provide insights into the mechanism of action of a novel pyrazolo[3,4-

d]pyrimidine Src inhibitor in human osteosarcoma. Molecular bioSystems. 2014;10(6):1305-12.

\section{Figures}

A

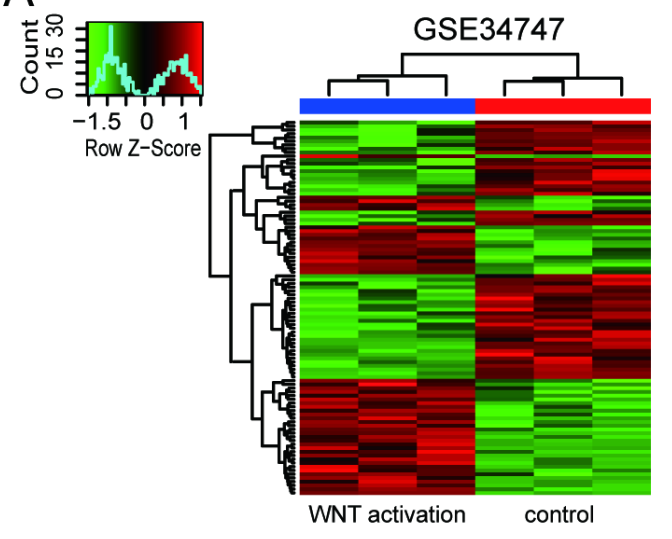

C detection of chemical stimulus involved in sensory perception detection of chemical stimulus

sensory perception of chemical stimulus system process

nervous system process

sensory perception

G protein-coupled receptor signaling pathway

signaling

signal transduction

cell communication

response to chemical

negative regulation of peptidase activity

multicellular organismal process

$\begin{array}{llcccccc}0 & 1 & 2 & 3 & 4 & 5 & 6 & 7\end{array}$

\section{cell periphery GO-Cellular Component}

\section{plasma membrane}

intrinsic component of membrane

membrane part

integral component of membrane

extracellular region

plasma membrane part

troponin complex

striated muscle thin filament

plasma membrane region

$\begin{array}{llllllllll}0 & 0.5 & 1 & 1.5 & 2 & 2.5 & 3 & 3.5 & 4 & 4.5\end{array}$
B

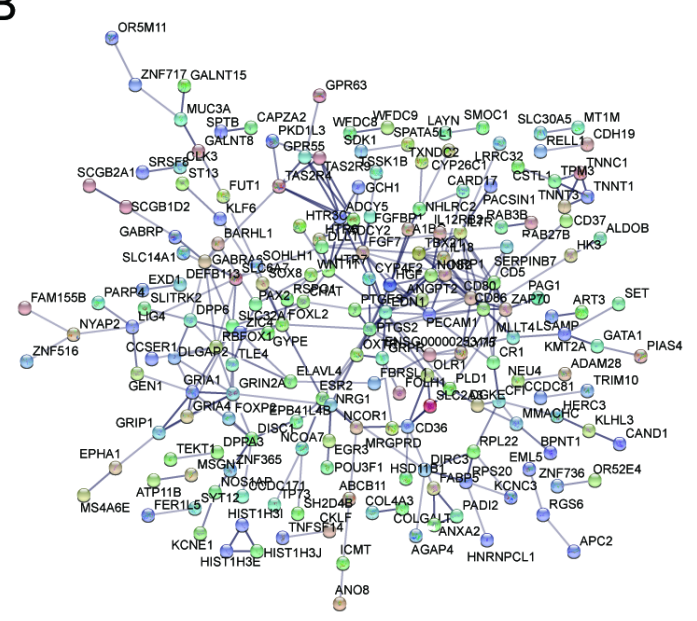

GO-Molecular Function

signaling receptor activity

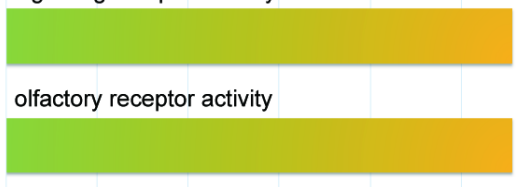

transmembrane signaling receptor activity

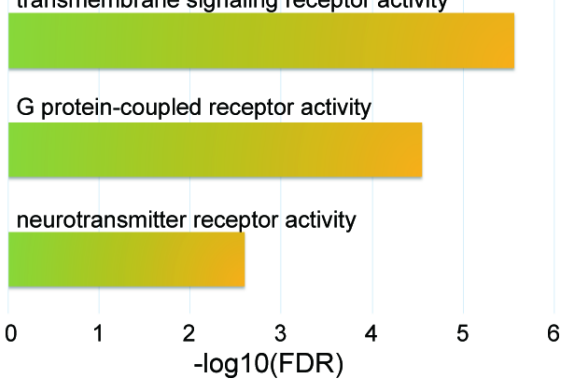

Reactome pathways

G alpha (s) signalling events

Olfactory Signaling Pathway

GPCR downstream signalling

Signaling by GPCR $-\log 10(F D R)$ 
Functional enrichment analysis of 562 DEGs in GSE34747. (A) Heat map of DEGs in GSE34747. Red color revealed the upregulated genes while the green color revealed the downregulated genes. (B) PPI network for DEGs was constructed using STRING. (C) GO enrichment and Reactome pathways enrichment of DEGs were analyzed by STRING. DEGs, differentially expressed genes. PPI, protein-protein interactions. GO, gene ontology. FDR, false discovery rate.

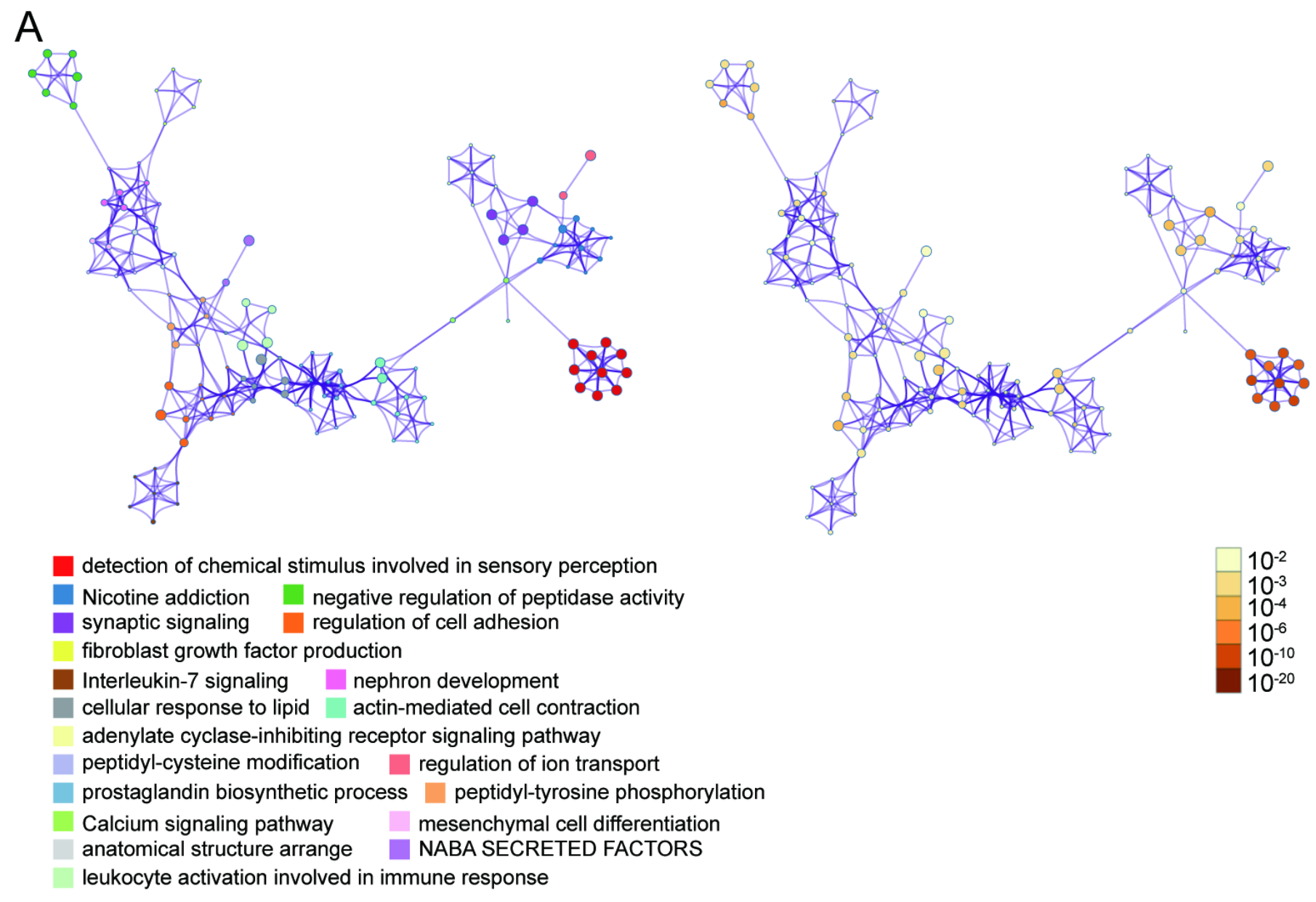

B

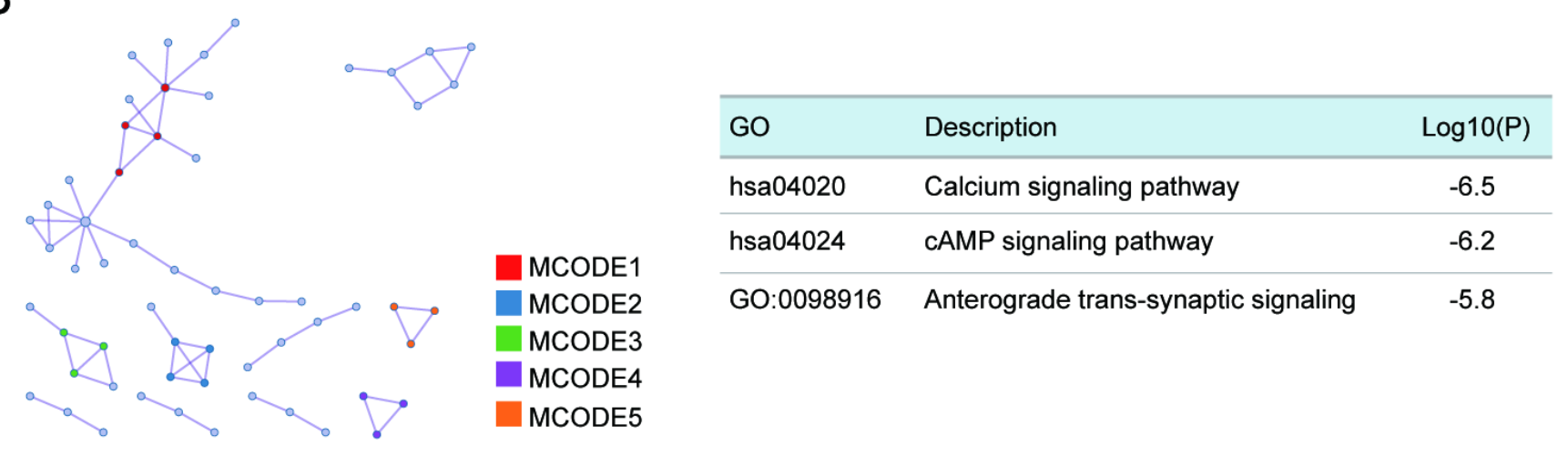

Figure 2

The analysis of GO enrichment, KEGG enrichment, and PPI network of 562 DEGs using Metascape. (A) The top 20 pathway and process enrichment was displayed using different colors. (B) The contruction of 
PPI network using Metascape. The top 3 MCODE were displayed. DEGs, differentially expressed genes. PPI, protein-protein interactions. GO, gene ontology. KEGG, Kyoto Encyclopedia of Genes and Genomes. MCODE, Molecular Complex Detection.

A
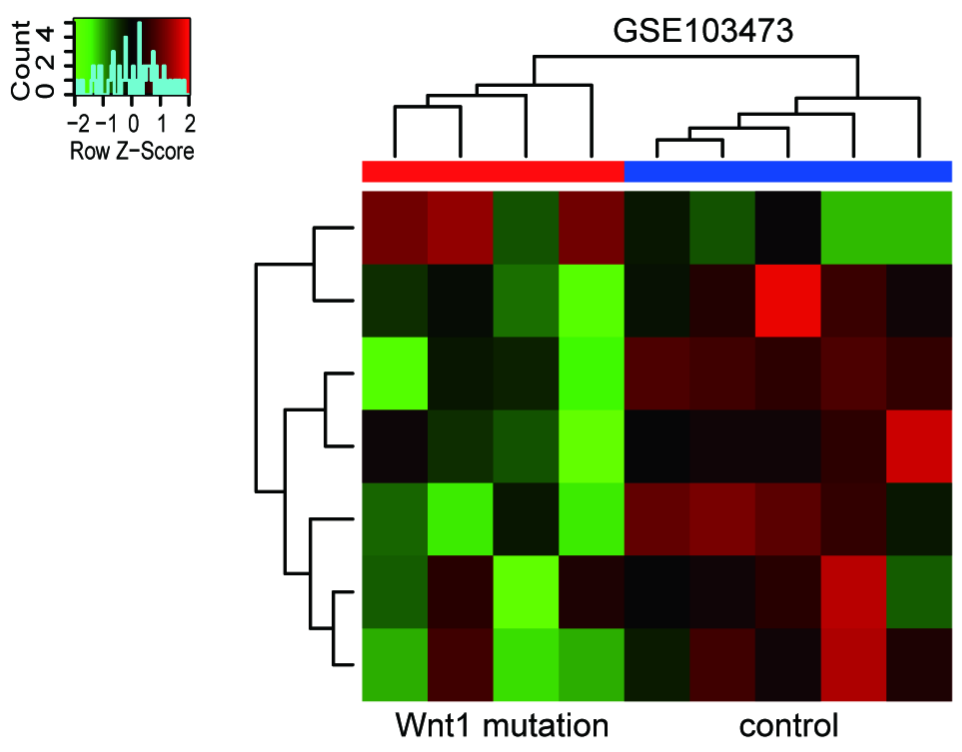

B

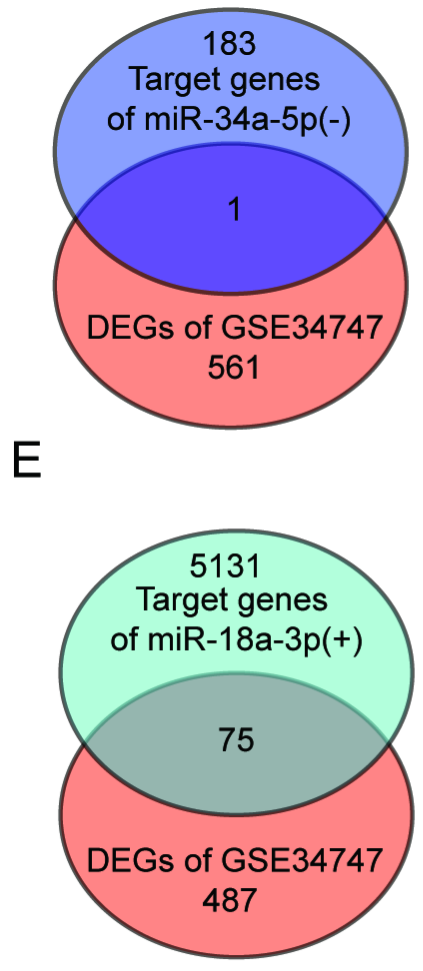

C

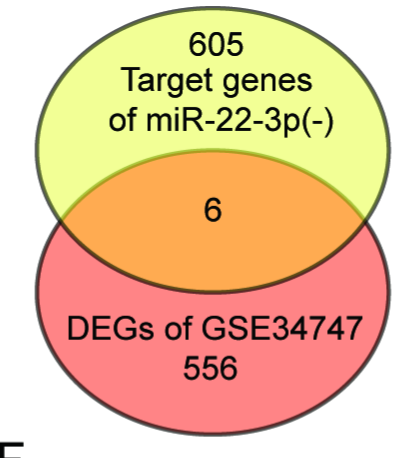

$\mathrm{F}$

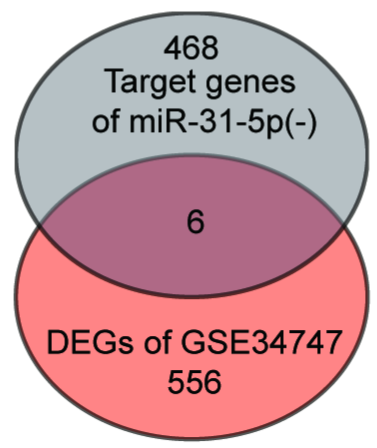

$\mathrm{D}$

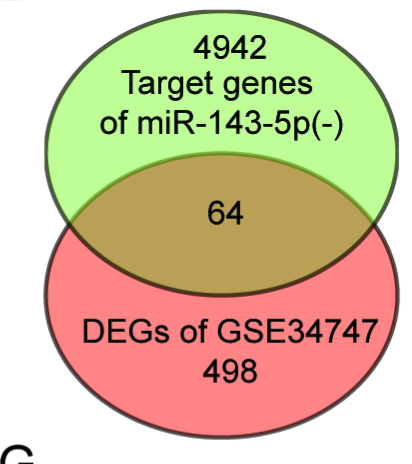

G

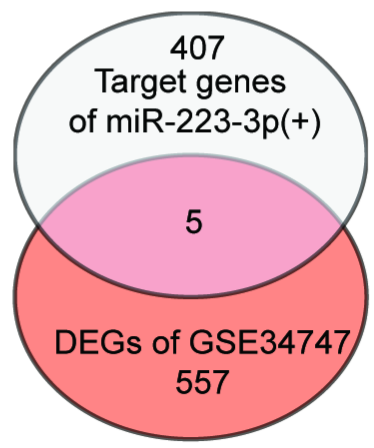

\section{Figure 3}

The target genes of top 6 differently expressed miRNAs were screened out using R software and Venny 2.1.0. (A) Heat map of differently expressed miRNAs in GSE103473 using R software. (B-G) Venny 2.1.0 
was applied to identify the target genes of miRNAs which also belonged to DEGs in GSE34747. DEGs, differentially expressed genes. -, low expression. +, high expression.
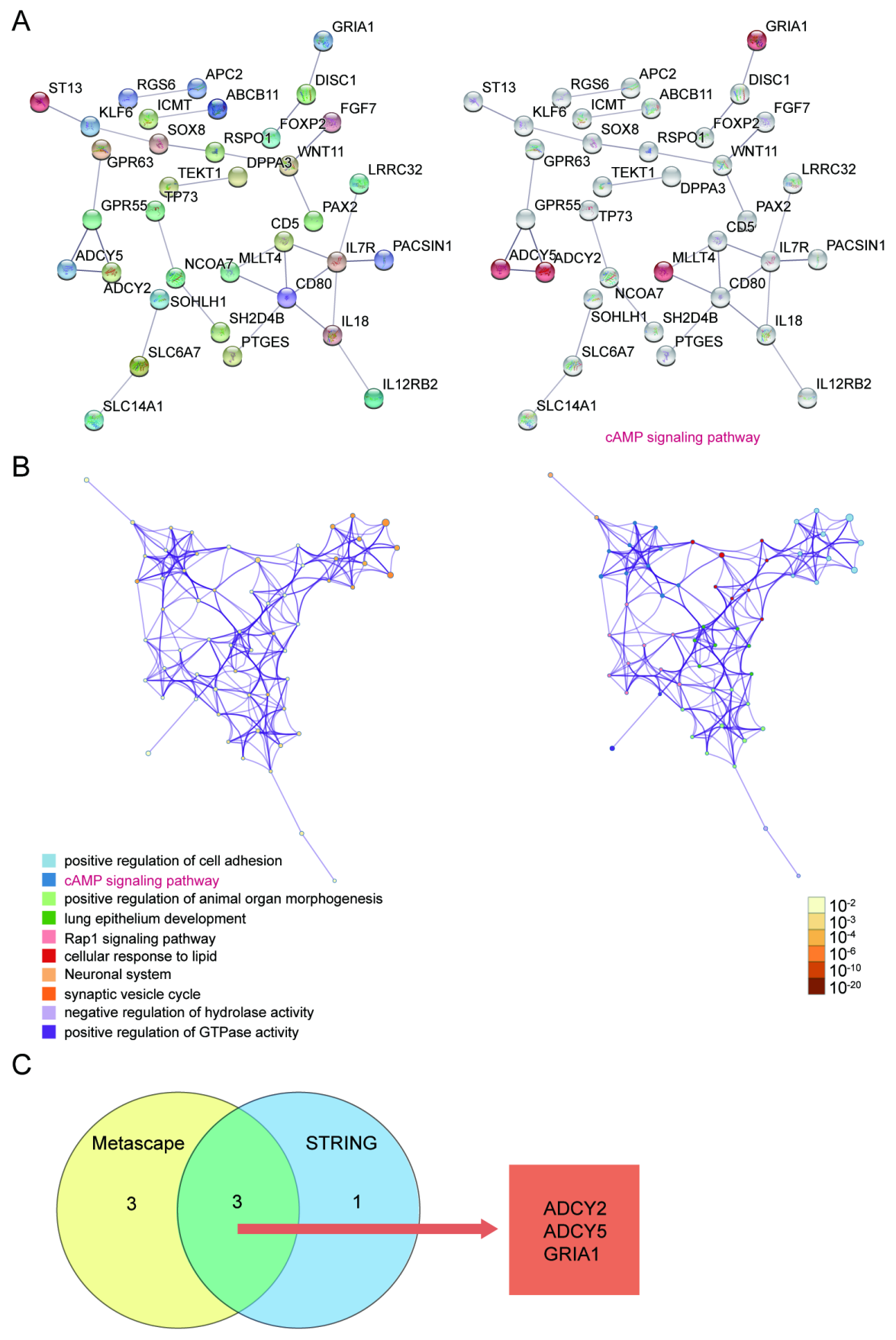

\section{Figure 4}

ADCY2, ADCY5, and GRIA1 were identified as key genes by STRING, Metascape and Venny 2.1.0. (A) The PPI network of 72 target genes of miR-18a-3p was constructed by STRING. The genes involving cAMP signaling pathway were list. (B) CAMP signaling pathway was the key pathway by Metascape analysis. 
Different colors represented different processes and pathways. (C) The common genes (ADCY2, ADCY5, and GRIA1) from Metascape and STRING involving cAMP signaling pathway by Venny 2.1.0. PPI, proteinprotein interactions. 